\title{
Joint CFO and Channel Estimation in OFDM-based Massive MIMO Systems
}

\author{
Hamed Hojatian \\ ECE Department \\ Isfahan University of Technology \\ Isfahan Iran 84156-83111 \\ h.hojatian@ec.iut.ac.ir \\ Mohammad Javad Omidi \\ ECE Department \\ Isfahan University of Technology \\ Isfahan Iran 84156-83111 \\ omidi@cc.iut.ac.ir
}

\author{
Hamid Saeedi-Sourck \\ EE Department \\ Yazd University \\ Yazd Iran 89158-18411 \\ saeedi@yazd.ac.ir \\ Arman Farhang \\ CONNECT, Trinity College Dublin, Ireland \\ farhanga@tcd.ie
}

\begin{abstract}
Estimation of carrier frequency offset (CFO) is a challenging task in practical systems specifically in the uplink of multiuser systems where multiple CFOs are present in the received signal. Massive MIMO as a multiuser technique has recently attracted a great deal of attention among researchers. However, to the best of our knowledge, there is no study looking into the joint estimation of CFOs and wireless channel in orthogonal frequency division multiplexing (OFDM) based massive MIMO systems. Therefore, in this paper, we propose joint estimation of multiple CFOs and the users' channel responses based on the maximum likelihood (ML) criteria in such systems. We propose to use the zadoff-chu (ZC) training sequences to reduce the implementation complexity. Additionally, utilization of $\mathrm{ZC}$ sequences for training simplifies the multidimensional grid search problem of estimating multiple CFOs and converts it into a set of line search problems, i.e., one line search problem per user. Also this sequence has a low peak to average ratio (PAPR). Finally, we show the efficacy of our proposed algorithm through numerical simulations.
\end{abstract}

\section{INTRODUCTION}

In the recent past, massive multiple input multiple output (MIMO), as a strong candidate technology for the fifth generation wireless networks $(5 \mathrm{G})$, has become the center of attention among many researchers in both industry and academia, [1]-[5]. Massive MIMO is a multiuser technology allowing simultaneous utilization of the available time-frequency resources by all the active users, [1]. This leads to a significant improvement in the capacity of the multiuser networks. In massive MIMO, the base station (BS) is equipped with a large number of antennas at the BS, i.e., in the order of hundreds, serving few tens of single antenna mobile terminals (MTs). Different users' signals at the BS are distinguished through the corresponding channel responses between each MT antenna and the BS antennas. As the number of the BS antennas grows large, the effects of both noise and multiuser interference (MUI) average out through utilization of the simplest detection

978-1-5090-3435-2/16/\$31.00 2016 IEEE methods such as maximum ratio combining (MRC), [1]. These assumptions are only valid when we have perfect knowledge of the channel responses at the base station and all the users are perfectly synchronized with the base station. However, in practice, synchronization errors appear in the received signal at the BS and can hamper the communication if they are not corrected.

Similar to [1], in this paper, we consider orthogonal frequency division multiplexing (OFDM) for data transmission. However, one of the main drawbacks of OFDM is its sensitivity to carrier frequency offset (CFO) especially in the uplink of multiuser networks where multiple CFOs are present in the received signal at the $\mathrm{BS},[6]-[8]$. This shortcoming is inherited to OFDM-based massive MIMO systems demanding accurate frequency synchronization. The CFOs are caused by the local oscillator misalignments and Doppler frequency shifts of different users due to the mobility. This breaks orthogonality among the subcarriers and leads to a great amount of inter-carrier interference (ICI), necessitating CFO estimation and/or correction before performing channel estimation and equalization.

In the uplink of the conventional orthogonal frequency division multiple access (OFDMA) systems, CFO estimation is a multidimensional grid search and hence very complex, [6], [8]. Additionally, correction of multiple CFOs in such systems is a computationally demanding task especially when the number of active users grows large, [8], [9]. The problem of CFO estimation and compensation in OFDM-based systems with spatial multiplexing, where a given subcarrier can be used by multiple users at the same time, become even more challenging. There exist a few works addressing the $\mathrm{CFO}$ and channel estimation problem in the literature. In [10], the authors propose a semi-blind method to simultaneously estimate multiple CFOs and channel responses in a multiuser MIMO setup. However, they only consider zero- 
padded OFDM (ZP-OFDM). A suboptimal method for CFO estimation has been proposed by using constant amplitude zero autocorrelation (CAZAC) training sequences in [11]. As an early work on CFO estimation in massive MIMO, the authors in [12] propose an maximum likelihood (ML)-based CFO estimator where they use an approximation for the purpose of complexity reduction. However, their solution requires a multidimensional grid search and thus is expected to have a high complexity. In [13], a pilot-based CFO estimation technique has been proposed for single carrier massive MIMO. However, the pilot sequence which has been used in this method leads to a high peak to average power ratio (PAPR). This requires linear power amplifier (PA) to work in its linear region which generally makes it power inefficient.

Based on the above discussion, the existing CFO estimation techniques in the literature either consider single carrier communications, [13] or a flat fading channel over the whole transmission band [12]. Hence, to the best of our knowledge, there is no study addressing CFO estimation and compensation problem in massive MIMO based on OFDM while considering wireless channel estimation. To this end, in this paper, we propose a joint estimation of multiple CFOs and the users' channel responses based on the ML criteria in the uplink of an OFDM-based massive MIMO system. To reduce the computational complexity of the $\mathrm{CFO}$ and Channel estimation, we propose using the zadoff-chu (ZC) training sequences. The choice of $\mathrm{ZC}$ sequences for training reduces the multidimensional grid search problem of multiple CFO estimation into a set of line search problems, i.e., one per user. Opposed to [11] where two OFDM symbols are utilized to transmit the $\mathrm{ZC}$ sequences and only $\mathrm{CFO}$ estimation is performed, in our solution the ZC sequences occupy only one OFDM symbol while enabling both CFO and channel estimation. Moreover, since the $\mathrm{ZC}$ sequences have a constant amplitude, such a choice for the training signals does not lead to the PAPR problem, [14], opposed to [13]. Our simulation results corroborate the effectiveness of the proposed $\mathrm{CFO}$ and channel estimation and compensation techniques in this paper. To further evaluate the performance of our proposed technique, we compare the bit error rate (BER) performance of our proposed technique with the fully synchronous case where perfect knowledge of the channel responses is available at the base station.

The rest of this paper is organized as follows. In Section II, we present the massive MIMO system model. Our proposed CFO and channel estimation algorithm together with the CFO correction and equalization is discussed in Section III. The performance of our proposed technique is evaluated in Section IV through simulation results. Finally, the conclusions of the paper are drawn in Section V.

Notation: Matrices, vectors and scalar quantities are denoted by boldface uppercase, boldface lowercase and normal letters, respectively. Superscripts $(.)^{\mathrm{H}},(.)^{\mathrm{T}}$ and $(.)^{-1}$ denote Hermitian, transpose, and the inverse of a matrix, respectively. $\mathbf{I}_{N}$ is an $N \times N$ identity matrix, $\mathcal{F}$ is the $N$-point discrete Fourier

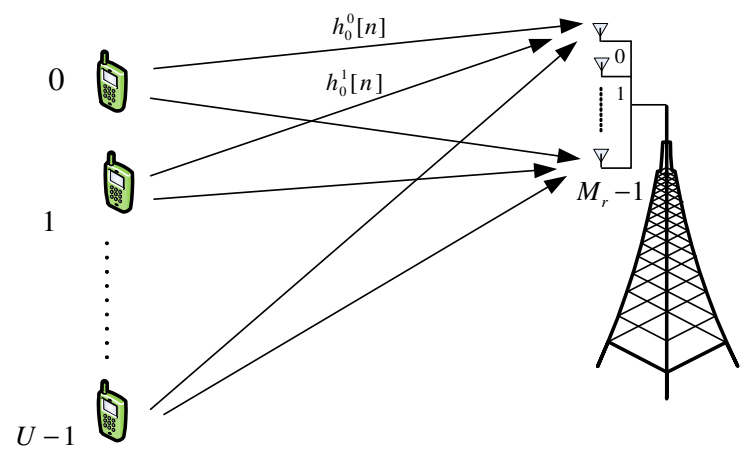

Fig. 1. Massive MIMO system setup.

transform (DFT) matrix and $\ominus$ denotes circular subtraction operation. Finally, $\mathbf{D}=\operatorname{diag}(\mathbf{a})$ is a diagonal matrix whose diagonal elements are formed by the elements of the vector $\mathbf{a}$.

\section{SySTEM MODEL}

We consider a single cell massive MIMO setup where $U$ single-antenna MTs are simultaneously communicating with $M_{\mathrm{r}}$ antennas at the $\mathrm{BS}$ in a time division duplexing (TDD) manner (Fig. 1). Throughout the paper, we consider $M_{\mathrm{r}}$ to be much larger than the number of users, i.e., $M_{\mathrm{r}} \gg U$. As Fig. 1 suggests, we assume a collocated antenna architecture where all the BS antennas are synchronized with respect to each other. Thus, the CFOs between each MT and all the $M_{\mathrm{r}}$ BS antennas have the same value. Independent frequencyselective channel responses are considered between the MT and BS antennas. We assume the channel length of $L>1$ for all the channel responses.

Let the time-domain discrete signal transmitted by the $u$ th MT denoted as $x^{u}[n]$ and $n$ the time-sample index within the range $-N_{\mathrm{cp}} \leq n \leq N-1$ where $N_{\mathrm{cp}}$ is the cyclic prefix (CP) length and $N$ is the total number of subcarriers. After removing $\mathrm{CP}$, the time domain discrete signal received at the $m$ th BS antenna can be denoted as

$$
y_{m}[n]=\sum_{u=0}^{U-1} \exp \left(\frac{j 2 \pi \varepsilon^{u} n}{N}\right) \sum_{\ell=0}^{L-1} h_{m}^{u}[\ell] x^{u}[n-\ell]+\nu_{m}[n],
$$

where $0 \leq n \leq N-1,-0.5<\varepsilon^{u}<0.5$ is the fractional CFO normalized to the subcarrier spacing, $\nu_{m}[n]$ is the zero-mean additive white Gaussian noise (AWGN) with the variance of $\sigma_{\nu}^{2} . h_{m}^{u}[n]$ is the channel impulse response between the $u$ th MT and the $m$ th BS antenna with length $L \leq N_{\text {cp. }}$.

According to (1), the received signal vector $\mathbf{y}_{m}=$ $\left[y_{m}[0], y_{m}[1], \cdots, y_{m}[N-1]\right]^{\mathrm{T}}$ at the BS antenna $m$ can be formulated as

$$
\mathbf{y}_{m}=\sum_{u=0}^{U-1} \boldsymbol{\Psi}^{u} \boldsymbol{\Lambda}^{u} \mathbf{h}_{m}^{u}+\boldsymbol{\nu}_{m}
$$


where $\boldsymbol{\nu}_{m}=\left[\nu_{m}[0], \nu_{m}[1], \ldots, \nu_{m}[N-1]\right]^{T}$ is the AWGN vector, the $N \times N$ phase rotation matrix $\boldsymbol{\Psi}^{u}$ is

$$
\boldsymbol{\Psi}^{u}=\operatorname{diag}\left[\exp \left(j \psi^{u}[0]\right), \cdots, \exp \left(j \psi^{u}[N-1]\right)\right],
$$

$\psi^{u}[i]=2 \pi \varepsilon^{u} i / N$, and the vector

$$
\mathbf{h}_{m}^{u}=\left[h_{m}^{u}[0], h_{m}^{u}[1], \cdots, h_{m}^{u}[L-1]\right]^{\mathrm{T}},
$$

includes the channel impulse response samples between the $u$ th MT antenna and the BS antenna $m$. The $N \times L$ matrix

$$
\boldsymbol{\Lambda}^{u}=\left[\begin{array}{cccc}
x^{u}[0] & x^{u}[N-1] & \cdots & x^{u}[N-L+1] \\
x^{u}[1] & x^{u}[0] & \cdots & x^{u}[N-L+2] \\
\vdots & \vdots & \ddots & \vdots \\
x^{u}[N-1] & x^{u}[N-2] & \cdots & x^{u}[N-L]
\end{array}\right]
$$

contains the first $L$ columns of a circulant matrix whose first column includes the first $N$ samples of the received signal at antenna $m$ after discarding the CP. Finally, (2) can be rearranged as

$$
\mathbf{y}_{m}=\boldsymbol{\Gamma} \tilde{\mathbf{h}}_{m}+\boldsymbol{\nu}_{m},
$$

where

$$
\boldsymbol{\Gamma}=\left[\boldsymbol{\Psi}^{0} \boldsymbol{\Lambda}^{0}, \boldsymbol{\Psi}^{1} \boldsymbol{\Lambda}^{1}, \cdots, \boldsymbol{\Psi}^{U-1} \boldsymbol{\Lambda}^{U-1}\right]_{N \times U L},
$$

and

$$
\tilde{\mathbf{h}}_{m}=\left[\left(\mathbf{h}_{m}^{0}\right)^{\mathrm{T}},\left(\mathbf{h}_{m}^{1}\right)^{\mathrm{T}}, \cdots,\left(\mathbf{h}_{m}^{U-1}\right)^{\mathrm{T}}\right]^{\mathrm{T}} .
$$

\section{Proposed Frequency Synchronization TECHNIQUE}

Frequency synchronization is a crucial task in the uplink transmission since the received signal at the BS is the combination of different users' signals affected by different wireless channels and CFOs [6]-[8]. In the following subsections, first a joint data-aided CFO and channel estimation is proposed, and then CFO compensation as well as channel equalization are discussed.

\section{A. Joint CFO and channel estimation}

In this subsection, we propose a joint $\mathrm{CFO}$ and channel estimation algorithm using $\mathrm{ZC}$ training sequences. To this end, we apply ML estimation criteria to equation (6) for the $m$ th antenna. We assume that the training signals transmitted from different MTs are known at the base station. Therefore, the logarithm of the conditional probability density function (pdf) of $\mathbf{y}_{m}$, given $\varepsilon_{m}$ and $\mathbf{h}_{m}$, can be written as

$$
\begin{aligned}
\ln p\left(\mathbf{y}_{m} \mid \varepsilon_{m}, \mathbf{h}_{m}^{u}\right) & =\Theta_{0}-\Theta_{1}\left[\mathbf{y}_{m}-\Gamma \mathbf{h}_{m}^{u}\right]^{\mathrm{H}} \\
& \times\left[\mathbf{y}_{m}-\boldsymbol{\Gamma} \mathbf{h}_{m}^{u}\right],
\end{aligned}
$$

where $\Theta_{0}=-N \ln \left(2 \pi \sigma_{\nu}^{2}\right) / 2, \Theta_{1}=1 / 2 \sigma_{\nu}^{2}$. By taking derivative of the log-likelihood function and setting it to zero, the estimation of the channel response betweem the $m$ th antenna and $u$ th MT can be obtained as

$$
\hat{\mathbf{h}}_{m}^{u}=\left(\boldsymbol{\Gamma}^{\mathrm{H}} \boldsymbol{\Gamma}\right)^{-1} \boldsymbol{\Gamma}^{\mathrm{H}} \mathbf{y}_{m} .
$$

Thus, by substituting (10) into (9), we have

$$
\begin{aligned}
\hat{\boldsymbol{\varepsilon}}_{m} & =\arg \max _{\varepsilon}\left\{\ln p\left(\mathbf{y}_{m} \mid \varepsilon_{m}\right)\right\} \\
& =\arg \max _{\varepsilon}\left\{\Theta_{0}-\Theta_{1} \times\left[\mathbf{y}_{m}-\boldsymbol{\Gamma}\left(\boldsymbol{\Gamma}^{\mathrm{H}} \boldsymbol{\Gamma}\right)^{-1} \boldsymbol{\Gamma}^{\mathrm{H}} \mathbf{y}_{m}\right]^{\mathrm{H}}\right. \\
& \left.\times\left[\mathbf{y}_{m}-\boldsymbol{\Gamma}\left(\boldsymbol{\Gamma}^{\mathrm{H}} \boldsymbol{\Gamma}\right)^{-1} \boldsymbol{\Gamma}^{\mathrm{H}} \mathbf{y}_{m}\right]\right\},
\end{aligned}
$$

where $\hat{\varepsilon}_{m}=\left[\hat{\varepsilon}_{m}^{0}, \hat{\varepsilon}_{m}^{1}, \cdots, \hat{\varepsilon}_{m}^{U-1}\right]$ is the estimated CFO vector on the $m$ th antenna at the BS whose elements are different users' CFOs with indices $u=0, \ldots, U-1$. By ignoring the terms irrelevant to $\varepsilon_{m}$ in (11), we have

$$
\hat{\varepsilon}_{m}=\arg \max _{\varepsilon}\left\{\mathbf{y}_{m}^{\mathrm{H}} \boldsymbol{\Gamma}\left(\boldsymbol{\Gamma}^{\mathrm{H}} \boldsymbol{\Gamma}\right)^{-1} \boldsymbol{\Gamma}^{\mathrm{H}} \mathbf{y}_{m}\right\} .
$$

The computational complexity of $\left(\boldsymbol{\Gamma}^{\mathrm{H}} \boldsymbol{\Gamma}\right)^{-1}$ may become too high in real-time applications. Hence, if we assume

$$
\Gamma^{\mathrm{H}} \boldsymbol{\Gamma} \approx c \mathbf{I},
$$

where $c$ is a constant value, the computational complexity of (12) is greatly reduced. Therefore, we propose to use ZC sequences, [15], for training

$$
\mathbf{z}[n]=\exp \left(\frac{j M \pi n^{2}}{N}\right), \quad n=0, \cdots, N-1,
$$

where $M$ is an integer parameter relatively prime to $N$ and $\mathbf{z}=[z[0], z[1], \cdots, z[N-1]]^{T}$. This sequence has good properties such as constant amplitude and zero autocorrelation for any circular shifts [14]. Each element of this sequence has an amplitude equal to one. The autocorrelation of $\mathrm{ZC}$ sequence satisfies

$$
R[k]=\sum_{n=0}^{N-1} \mathbf{z}[n] \mathbf{z}^{*}[n \ominus k]= \begin{cases}N & k=0, \\ 0 & k \neq 0 .\end{cases}
$$

This means that the ZC sequence is orthogonal to all non-zero circular shifts. Let $\mathbf{Z}$ be a circulant matrix with the first column equal to $[z(0), z(1), \cdots, z(N-1)]^{T}$. The autocorrelation property of the $\mathrm{ZC}$ sequence can be rewritten in an equivalent matrix form as

$$
\mathbf{Z}^{\mathrm{H}} \mathbf{Z}=N \mathbf{I} .
$$

According to (15), we can generate an orthogonal training sequence for each MT with respect to the other MTs by circular shifting the sequence in time domain. Denoting the training sequences as $\zeta^{u}=\left[\zeta^{u}[0], \ldots, \zeta^{u}[N-1]\right]^{T}$ for $0 \leq$ $u \leq U-1$, we define the training sequence used at the $u$ th MT as $\boldsymbol{\zeta}^{u}=\mathbf{z}\left\langle Q^{u}\right\rangle$, where

$\mathbf{z}\left\langle Q^{u}\right\rangle=\left[z\left[N-Q^{u}\right], \cdots, z[N], z[0], \cdots, z\left[N-Q^{u}-1\right]\right]^{\mathrm{T}}$,

is a circular-shifting of $\mathbf{z}$ to the right direction by $Q^{u}$ samples, $0 \leq Q^{u} \leq N-1$. For example, for the first MT we use the prime $\mathrm{ZC}$ sequence; that is, $\boldsymbol{\zeta}^{1}=\mathbf{z}$ where $Q^{1}=0$. Theoretically, applying orthogonal sequences as the training 
sequences achieves the mutual interference free condition in the absence of CFO. However, in the presence of CFOs, mutual interference is inevitable and the estimation performance is severely degraded.

By applying the proposed training sequences, $\boldsymbol{\Gamma}^{\mathrm{H}} \boldsymbol{\Gamma} \approx N \mathbf{I}$ can be retained and the reduced complexity ML estimator in (12) reduces to

$$
\hat{\varepsilon}_{m}=\arg \max _{\varepsilon}\left\{\mathbf{y}_{m}^{\mathrm{H}} \boldsymbol{\Gamma} \boldsymbol{\Gamma}^{\mathrm{H}} \mathbf{y}_{m} / N\right\} .
$$

Compared with (12), (18) has a lower computational complexity and the estimation of $\varepsilon_{m}$ for the $u$ th MT can be obtained by inserting (7) into (18) as

$$
\begin{aligned}
\hat{\boldsymbol{\varepsilon}}_{m}= & \arg \max _{\varepsilon}\left\{\mathbf{y}_{m}^{\mathrm{H}} \boldsymbol{\Psi}^{0} \boldsymbol{\Lambda}^{0}\left(\boldsymbol{\Lambda}^{0}\right)^{\mathrm{H}}\left(\boldsymbol{\Psi}^{0}\right)^{\mathrm{H}} \mathbf{y}_{m}+\cdots\right. \\
& \left.+\mathbf{y}_{m}^{\mathrm{H}} \boldsymbol{\Psi}^{U-1} \boldsymbol{\Lambda}^{U-1}\left(\boldsymbol{\Lambda}^{U-1}\right)^{\mathrm{H}}\left(\boldsymbol{\Psi}^{U-1}\right)^{\mathrm{H}} \mathbf{y}_{m}\right\} .
\end{aligned}
$$

Therefore, CFO estimation for all the users can be performed independently. Consequently, the CFO estimate for the $u$ th MT on the $m$ th antenna is obtained as

$$
\hat{\varepsilon}_{m}^{u}=\arg \max _{\varepsilon}\left\{\mathbf{y}_{m}^{\mathrm{H}} \boldsymbol{\Psi}^{u} \boldsymbol{\Lambda}^{u}\left(\boldsymbol{\Lambda}^{u}\right)^{\mathrm{H}}\left(\boldsymbol{\Psi}^{u}\right)^{\mathrm{H}} \mathbf{y}_{m}\right\} .
$$

Based on 20, multidimensional grid search problem for $\mathrm{CFO}$ estimation in (11) converts into a set of line search problems, i.e., one per user. Finally, the estimated CFO value for the desired user $u$ is achieved by averaging over all the estimated $\mathrm{CFO}$ values on different antennas, i.e.,

$$
\hat{\varepsilon}^{u}=\frac{1}{M_{r}} \sum_{m=0}^{M_{r}-1} \hat{\varepsilon}_{m}^{u},
$$

As it will be shown in section IV, (21) improves the CFO estimation accuracy as the number of BS antennas increases. According to (10), using the $\mathrm{ZC}$ pilot sequences, we can approach to $\boldsymbol{\Gamma}^{\mathrm{H}} \boldsymbol{\Gamma} \approx N \mathbf{I}$ for channel estimation too. Then the corresponding channel impulse response estimator on the $m$ th antenna is given by

$$
\hat{\mathbf{h}}_{m}=\boldsymbol{\Gamma}^{\mathrm{H}} \mathbf{y}_{m} / N
$$

By substitution of (7) into (22), we have

$$
\hat{\mathbf{h}}_{m}^{u}=\left(\left(\boldsymbol{\Lambda}^{u}\right)^{\mathrm{H}}\left(\hat{\boldsymbol{\Psi}}^{u}\right)^{\mathrm{H}} \mathbf{y}_{m}\right) / N,
$$

where $\hat{\mathbf{h}}_{m}^{u}$ is the estimated channel impulse response between the $u$ th MT antenna and the BS antenna $m . \hat{\boldsymbol{\Psi}}^{u}$ is an estimation of $\boldsymbol{\Psi}^{u}$ given in (3).

\section{B. CFO compensation at the receiver}

After CFO and channel estimation, the next goal of this paper is to present a receiver structure to recover transmitted symbols by $\mathrm{CFO}$ compensation and channel equalization for different users. In this section a trivial CFO compensation is discussed.

It can be shown that the received signal in presence of CFOs and wireless channel at the BS on the $m$ th antenna in (2) can be rewritten as

$$
\mathbf{y}_{m}=\sum_{u=0}^{U-1} \boldsymbol{\Psi}^{u} \mathcal{F}^{\mathrm{H}} \mathcal{H}_{m}^{u} \mathbf{s}^{u}+\boldsymbol{\nu}_{m}
$$

where $\mathbf{s}^{u}=\left[s^{u}(0), s^{u}(1), \cdots, s^{u}(N-1)\right]^{\mathrm{T}}$ is the symbol vector transmitted from the $u$ th MT and $\mathcal{H}_{m}^{u}=\operatorname{diag}\left(\overline{\mathbf{h}}_{m}^{u}\right)$ is a diagonal matrix including the channel frequency response between the $u$ th MT antenna and the $m$ th BS antenna, where

$$
\overline{\mathbf{h}}_{m}^{u}=\left[\bar{h}_{m}^{u}(0), \bar{h}_{m}^{u}(1), \cdots, \bar{h}_{m}^{u}(N-1)\right]^{\mathrm{T}},
$$

is $N \times 1$ vector which includes the samples from the $N$-point DFT of the channel impulse response.

A trivial CFO compensation may be performed for each user separately. Let us define

$$
\hat{\boldsymbol{\Psi}}^{-k}=\operatorname{diag}\left[\exp \left(-j \hat{\psi}^{k}[0]\right), \cdots, \exp \left(-j \hat{\psi}^{k}[N-1]\right)\right],
$$

where $\hat{\psi}^{k}[i]=2 \pi \hat{\varepsilon}^{k} i / N$. The aligned received signal vector for the $k$ th user is

$$
\hat{\mathbf{y}}_{m}^{k}=\hat{\boldsymbol{\Psi}}^{-k} \mathbf{y}_{m}=\hat{\boldsymbol{\Psi}}^{-k} \sum_{u=0}^{U-1} \boldsymbol{\Psi}^{u} \mathcal{F}^{\mathrm{H}} \mathcal{H}_{m}^{u} \mathbf{s}^{u}+\hat{\boldsymbol{\Psi}}^{-k} \boldsymbol{\nu}_{m} .
$$

Assuming $\hat{\varepsilon}^{k}=\varepsilon^{k}, \hat{\boldsymbol{\Psi}}^{-k} \boldsymbol{\Psi}^{k}=\mathbf{I}$, we have

$$
\hat{\mathbf{y}}_{m}^{k}=\mathcal{F}^{\mathrm{H}} \mathcal{H}_{m}^{k} \mathbf{s}^{k}+\hat{\boldsymbol{\Psi}}^{-k} \sum_{\substack{u=0 \\ u \neq k}}^{U-1} \boldsymbol{\Psi}^{u} \mathcal{F}^{\mathrm{H}} \mathcal{H}_{m}^{u} \mathbf{s}^{u}+\hat{\boldsymbol{\Psi}}^{-k} \boldsymbol{\nu}_{m}
$$

Hence, the desired term is $\mathcal{F}^{\mathrm{H}} \mathcal{H}_{m}^{k} \mathbf{s}^{k}$ and other terms can be assumed as interference and absorbed into the noise vector. As a result, the received signal vector after DFT is

$$
\hat{\mathbf{r}}_{m}^{k}=\mathcal{F} \hat{\mathbf{y}}_{m}^{k}=\mathcal{H}_{m}^{k} \mathbf{s}^{k}+\boldsymbol{\nu}_{m}^{\prime} \text {. }
$$

where $\hat{\mathbf{r}}_{m}^{k}=\left[\hat{r}_{m}^{k}(0), \hat{r}_{m}^{k}(1), \cdots, \hat{r}_{m}^{k}(N-1)\right]^{\mathrm{T}}$. It is worth mentioning that we separately compensate the effect of $\mathrm{CFO}$ for each user.

Finally, symbol estimation is performed in the subcarrier level. Let us define the received signal samples on the $i$ th subcarrier over all antennas for the $k$ th user as

$$
\hat{\mathbf{r}}^{k}(i)=\left[\hat{r}_{0}^{k}(i), \hat{r}_{1}^{k}(i), \cdots, \hat{r}_{M_{\mathrm{r}}-1}^{k}(i)\right]^{\mathrm{T}} .
$$

The estimated samples transmitted on the $i$ th subcarrier for the $k$ th user using matched filter detector are obtained as [16]

$$
\hat{\mathbf{s}}^{k}(i)=\frac{\left(\mathbf{w}_{i}^{k}\right)^{\mathrm{H}}}{\left\|\mathbf{w}_{i}^{k}\right\|^{2}} \hat{\mathbf{r}}^{k}(i),
$$

where $\mathbf{w}_{i}^{k}=\left[\bar{h}_{0}^{k}(i), \bar{h}_{1}^{k}(i), \cdots, \bar{h}_{M_{r}-1}^{k}(i)\right]^{\mathrm{T}}$.

\section{Simulation Results}

In this section, computer simulations are performed to evaluate the performance of the proposed joint $\mathrm{CFO}$ and channel estimator at the BS. We assume 4 MTs with random $\mathrm{CFO}$ values uniformly distributed within the range $-0.5<\varepsilon^{u}<0.5$ communicating with $M_{\mathrm{r}}=100$ antennas simultaneously at the BS unless otherwise stated. Each MT uses OFDM with $N=64$ subcarriers and the length of $\mathrm{CP}$ is $N / 4=16$. The channel gain remains unchanged during an OFDM symbol interval. Also we use extended typical urban (ETU) channel model [17]. Furthermore, the signals from MTs are assumed to have the same average power at each antenna. 


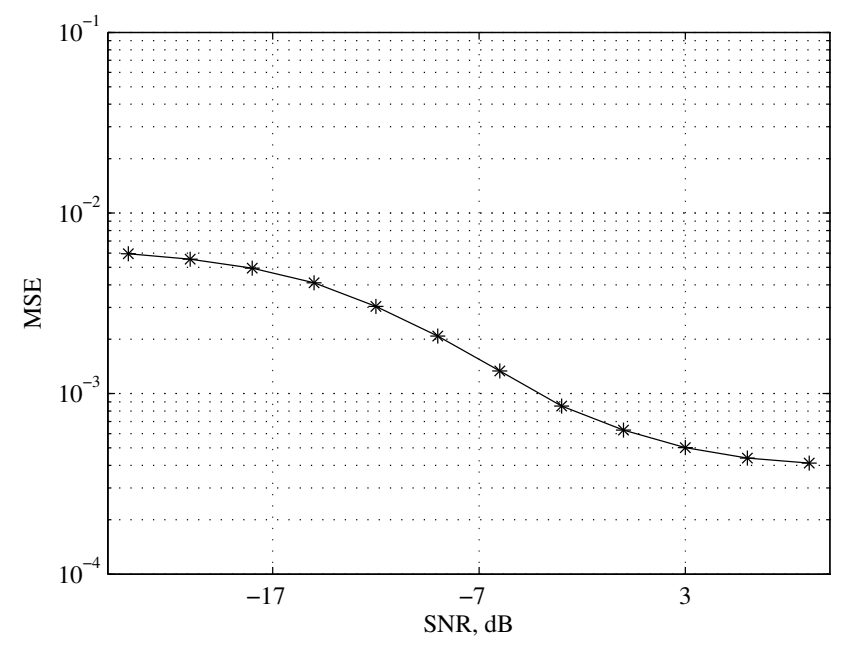

Fig. 2. MSE of $\mathrm{CFO}$ estimation with $\mathrm{CFO}$ vector $\varepsilon=$ $[0.1,-0.1,0.05,-0.05]$.

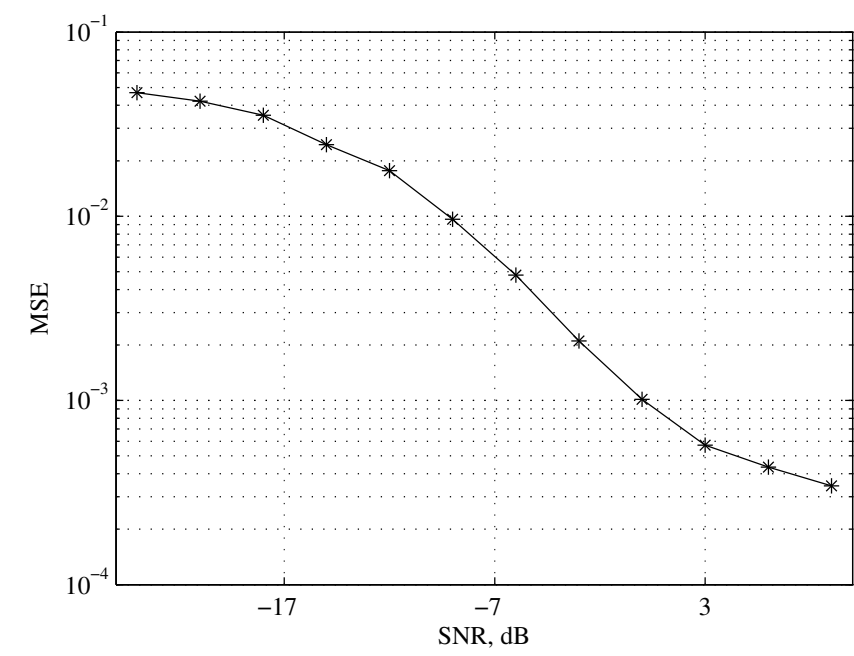

Fig. 3. MSE of the CFO estimation uniformly distributed in $-0.5<\varepsilon^{u}<$ 0.5 .

Fig. 2 shows mean square error (MSE) for constant CFO values $[0.1,-0.1,0.05,-0.05]$. Also MSE of random $\mathrm{CFO}$ values is presented in Fig. 3. As shown in these figures, the performance of the proposed estimator is improved as well as SNR is increased.

Fig. 4 shows channel estimation MSE of our proposed algorithm. In this figure, the performance of channel estimator are compared in two situations. In first case, we consider perfect knowledge of CFO values, while in the second case the $\mathrm{CFO}$ values are also estimated. As expected, the performance of channel estimation with perfect knowledge of CFOs has a superior performance than the case where the CFO values are estimated. However, the performance of the second case is comparable with that of perfectly known CFOs case.

The bit error rate (BER) performance of our proposed CFO

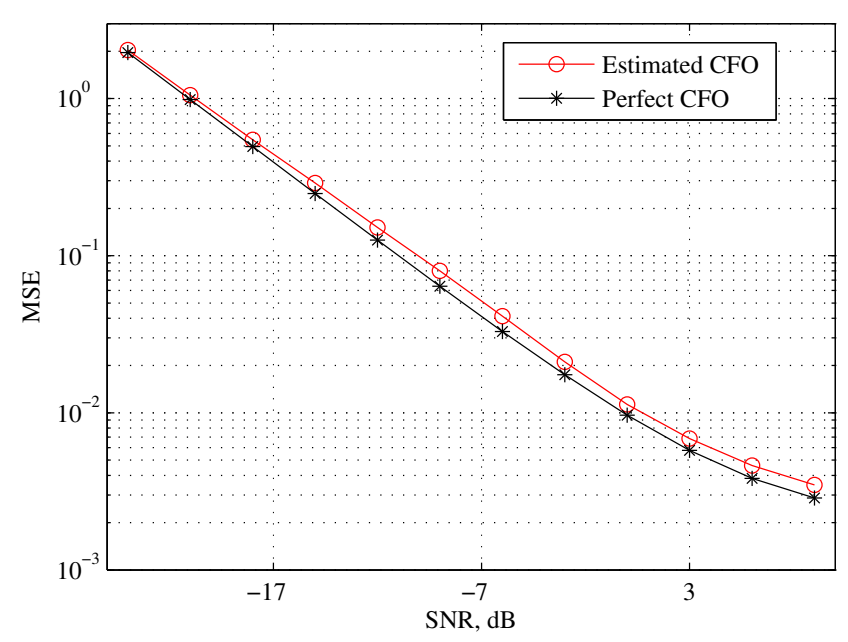

Fig. 4. MSE of channel estimation with perfect $\mathrm{CFO}$ and estimated CFO.

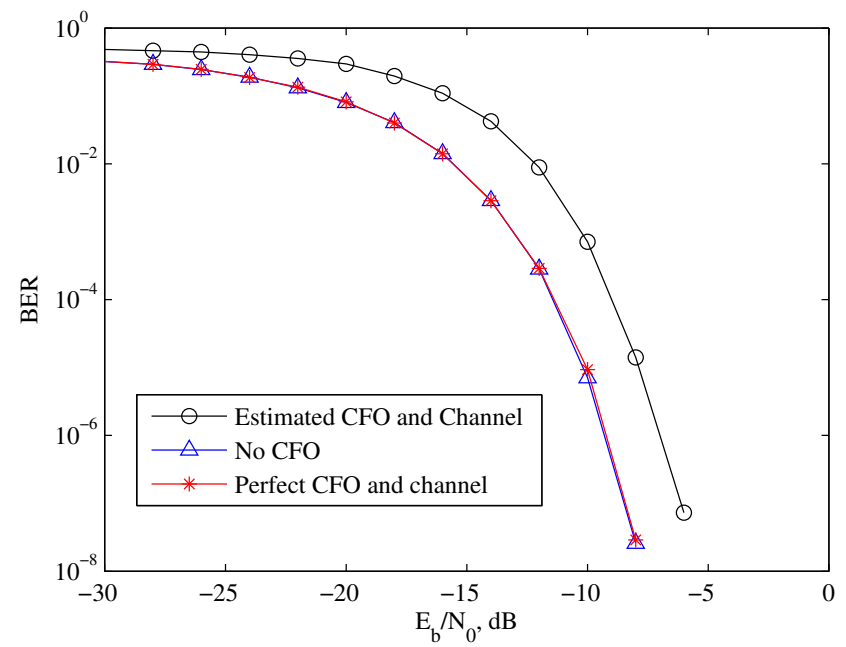

Fig. 5. BER performance of the proposed CFO estimation compared with the perfect CFO estimation and a system without CFO.

and channel estimation techniques are shown in Fig. 5. Fig. 6 presents the MSE performance of our proposed CFO estimation technique as a function of different $\mathrm{CFO}_{\max }$ ranges, where $\mathrm{CFO}$ values for all the users are assumed uniformly distributed within the range $-\mathrm{CFO}_{\max }<\varepsilon^{u}<\mathrm{CFO}_{\max }$ for each point in the curve and $E_{\mathrm{b}} / N_{0}=0 \mathrm{~dB}$. As shown in this figure, MSE is degraded with the increase of $\mathrm{CFO}_{\max }$. This is due to the fact that condition (13) is not valid for large CFO values.

Finally, Fig. 7 shows the MSE performance of our proposed estimation algorithm as a function of the number of BS antennas at $E_{\mathrm{b}} / N_{0}=0 \mathrm{~dB}$. As the number of BS antennas is increased, the CFO estimates become more accurate. For the number of BS antennas larger than 64, MSE is approximately constant. Consequently, the CFO estimation may be performed at a limited number ofBS antennas to reduce the computational 


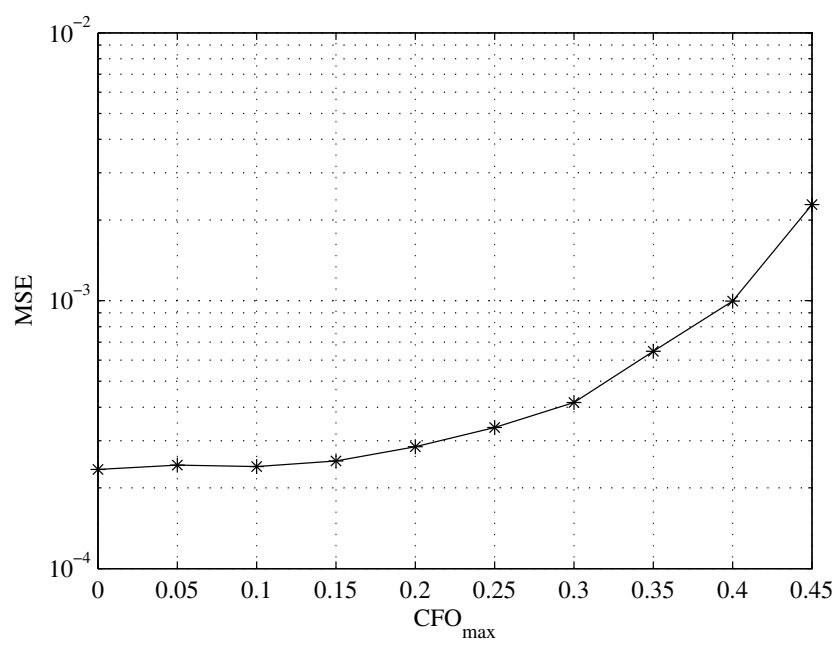

Fig. 6. MSE performance of our proposed $\mathrm{CFO}$ estimation technique as a function of different maximum $\mathrm{CFO}$ ranges.

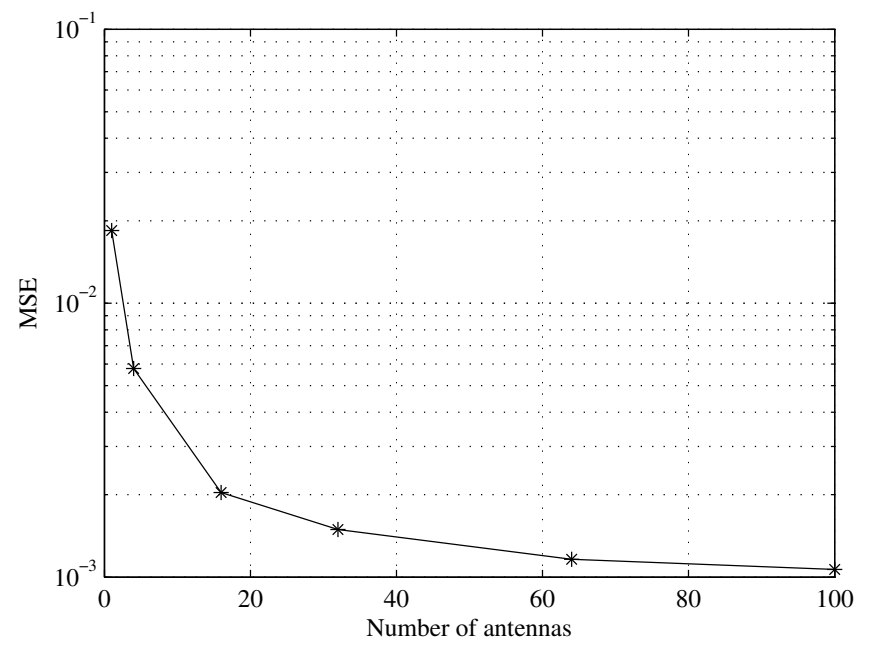

Fig. 7. MSE of CFO estimation versus the number of BS antennas.

burden of the system.

\section{CONClusion}

Massive MIMO has recently attracted a great deal of attention among researchers. In this paper, we proposed a joint estimation of multiple CFOs and the users' channel responses based on the ML criteria. We proposed to use the $\mathrm{ZC}$ training sequences as a low PAPR sequence to reduce the implementation complexity. Utilization of ZC sequences for training simplified the multidimensional grid search problem of estimating multiple CFOs and converts it into a set of line search problems. CFO estimation for each user was estimated on each antenna distinctly. Also the performance of CFO estimation was improved through averaging over part of antennas. Finally, we evaluated the efficiency of the proposed algorithm through numerical simulations.

\section{REFERENCES}

[1] T.L. Marzetta, "Noncooperative Cellular Wireless with Unlimited Numbers of Base station Antennas," IEEE Trans. on Commun., vol.9, no. 11, pp. 3590-3600, November 2010.

[2] E.G. Larsson, F. Tufvessom, O. Edfors, and T.L. Marzetta, "Massive MIMO for Next Generation Wireless System," IEEE Commun. Mag., vol. 52, no. 2, pp. 186-195, February 2014.

[3] H.Q. Ngo, E.G. Larsson, and T.L. Marzetta, "Energy and spectral efficiency of very large multi user MIMO systems," IEEE Trans. on Commun., vol. 61, no. 4, pp. 1436-1449, Apr. 2013.

[4] J. Hoydis, S. ten Brink, and M. Debbah, "Massive MIMO in the UL/DL of cellular networks: How many antennas do we need?" IEEE Journal on Selected Areas in Communications, vol. 31, no. 2, pp. 160-171, 2013.

[5] F. Rusek, D. Persson, B.K. Lau, E.G. Larsson, T.L. Marzetta, O. Edfors, F. Tufvesson, "Scaling up MIMO: Opportunities and challenges with very large arrays", IEEE Signal Process. Mag., vol. 30, no. 1, pp. 40-60, 2013.

[6] M.O. Pun, M. Morelli, and C.C.J. Kuo, "Multi- Carrier Techniques for Broadband Wireless Communications: A Signal Processing Perspective", Imperial College Press, 2007.

[7] P.H. Moose, "A technique for orthogonal frequency-division multiplexing frequency offset correction," IEEE Trans. on Commun., vol. 42, no. 10 , pp. 2908-2914, Oct. 1994.

[8] B.M. Morelli, C.C.J. Kuo, and M.N. Pun, "Synchronization techniques for orthogonal frequency division multiple access (OFDMA): A tutorial review, " proc. of the IEEE, vol. 95, no. 7, pp. 1394-1427, July 2007.

[9] A. Farhang and N. Marchetti and L. E. Doyle and B. FarhangBoroujeny,"Low complexity CFO compensation in uplink OFDMA systems with receiver windowing," IEEE Trans. on Signal Process., vol. 63 , no. 10, pp. 2546-2558, May 2015.

[10] Y. Zeng, R. A. Leyman. and T. S. Ng, "Joint semiblind frequency offset and channel estimation for multiuser MIMO-OFDM uplink," IEEE Trans. on Commun., vol. 55, no. 12, pp. 2570-2578, 2007.

[11] Y. Wu , J. W. M. Bergmans and S. Attallah, "Carrier frequency offset estimation for multiuser MIMO OFDM uplink using CAZAC sequence: performance and sequence optimization," EURASIP Journal on wirel. Commun. and Network., vol.2011, Article ID: 570680.

[12] H. Cheng and E. Larsson "Some Fundamental Limits on Frequency Synchronization in massive MIMO," in Signals, Systems and Computers, 2013 Asilomar Conference on, Nov 2013, pp. 1213-1217

[13] S. Mukherjee and S. K. Mohammad, "Low-Complexity CFO Estimation for Multi-User Massive MIMO Systems," submitted to Submitted to IEEE Globecom 2015, Available. https://arxiv.org/abs/1504.05657.

[14] G.C. Priya, and M. Suganthi, "Combined PAPR reduction and frequency offset estimation using precoded Zadoff-Chu OFDM in WLAN system “, 3rd IEEE International Conference on Computer Science and Information Technology (ICCSIT), 2010 , vol. 3, oo. 461-465.

[15] D. Chu,"Polyphase codes with good periodic correlation properties.", IEEE Trans. Inf. Theory, vol. 18, no. 4, pp. 531-532, July 1972.

[16] L. Lu, G.Y. Li, A.L. Swindlehurst, A. Ashikhmin, R. Zhang, "An Overview of Massive MIMO: Benefits and Challenges" , IEEE Journal of Selected Topics in Signal Processing, vol. 8, no. 5, pp. 742-758, Oct. 2014.

[17] W. B. Yang and M. Souryal, "LTE physical layer performance analysis", National institute of standard and technology, U.S. Department of Commerce, http://dx.doi.org/10.6028/NIST.IR.7986, May 2014. 